\title{
LE COMPETENZE AVANZATE DEL TECNICO SANITARIO DI RADIOLOGIA MEDICA
}

\author{
Dott. Di Lascio Antonio
}

Tsrm specialista in: Aspetti legali, forensi ed assicurativi delle professioni sanitarie, Rischio clinico in sanità, Amministratore di sistema, ASL Salerno - P.O. di Vallo della Lucania UOC di Emodinamica

KEYWORDS: TSRM, competenze avanzate, cardiologia interventistica, medicina nucleare, contratto collettivo nazionale, professioni sanitarie, ordine TSRM PSTRP

\section{ABSTRACT}

Il profilo del TSRM, è mutato è mutato, nel corso degli anni, fino ad esercente la "professione sanitaria", individuata sulla base di un preciso profilo legislativo (L. 31 gennaio 983 n. 25, DM 26 settembre 1994 n. 746 del 1994, L. 10 agosto 2000 n. 251), con l'affermazione, dello status di "Professione intellettuale" (art. 2229 cc, l. $42 / 19991$ e l. 251/2000), come per tutte le altre professioni sanitarie, adesso ordinate e sottoposte alla vigilanza del Ministero della Salute, a seguito dell'entrata in vigore della L. 3/2018.

L'evoluzione organizzativa, professionale, la collaborazione multiprofessionale richiesta, lo sviluppo tecnologico e i progressi della ricerca scientifica in ambito clinico-sanitario, ha comportato per le professioni sanitarie, ed in particolare per il TSRM, l'espletamento di alcune competenze avanzate. Lo scopo del presente lavoro è illustrare l'attuale posizione giuridica del TSRM e presentare alcune esperienze, in cui, il TSRM è chiamato a svolgere cosid-

${ }^{1}$ Art. 1 della L. 42/1999 "il campo proprio di attività e di responsabilità delle professioni sanitarie ... è determinato dai contenuti dei decreti ministeriali istitutivi dei relativi profili professionali e degli ordinamenti didattici dei rispettivi corsi di diploma universitario e di formazione post-base nonché degli specifici codici deontologici".

\section{INTRODUZIONE}

L'attività professionale nel corso di questi ultimi anni ha richiesto ai professionisti maggiori competenze, in termini di valore, qualità, conoscenze, doti (professionali e personali), capacità ed abilità, da questi possedute, con il passaggio da logiche di ausiliarietà, basate su mansioni ${ }^{1}$ di complemento, a favore, di ambiti di autonomia, con la conseguenza di rispondere, in modo completo e pertinente, della propria attività (sotto il profilo professionale e di rischio), complice anche un nuovo assetto formativo, voluto per le professioni sanitarie.

Il profilo del Tecnico sanitario di radiologia medica (TSRM), è passato da assistente ai "gabinetti radiologici", ad essere, prima, esercente di "arte ausiliaria" (RD 27 luglio 1934 n. 1265, legge 4 agosto 1965 n. 1103), ed infine, quale esercente la "professione sanitaria", individuata sulla base di un preciso profilo legislativo (L. 31 gennaio 983 n. 25, DM 26 settembre 1994 n. 746 del 1994, L. 10 agosto 2000 n. 251), secondo un percorso didattico-formativo definito, a livello ministeriale, che culmina, previo superamento dell'esame di abilitazione, con l'acquisizione del titolo di Tecnico sanitario di radiologia medica e la con- sequenziale affermazione, dello status di "Professioni intellettuali" (art. $2229 \mathrm{cc}, 1.42 / 1999^{2}$ e 1. 251/2000). Discorso, naturalmente valido e sovrapponibile, per tutte le altre professioni sanitarie, adesso ordinate e sottoposte alla vigilanza del Ministero della Salute, a seguito dell'entrata in vigore della L. 3/2018, che ha segnato un importante traguardo attraverso il pieno riconoscimento del contributo che negli anni, hanno profuso, per la costruzione e crescita del SSN e per la promozione della salute.

Tale impegno, accompagnato dalla collaborazione multiprofessionale, dallo sviluppo tecnologico e dai progressi della ricerca scientifica in ambito clinicosanitario necessità oggi di essere valorizzato e capitalizzato.

\section{METODOLOGIA E MATERIALI}

Per l'esecuzione delle attività tecnico-assistenziali sulla persona ${ }^{3}$, il Tecnico sanitario di radiologia medica, è fornito, di un'adeguata istruzione, informazione e formazione, sia teorica che pratica ${ }^{4}$ ed abilitato, secondo legge, ad operare in un campo riconosciuto di specializzazione ${ }^{5}$, quali sono, i tipici aspetti pratici delle procedure medico-radiologiche e quelli

${ }^{1}$ Elencazione di compiti e attribuzioni ai quali l'esercizio professionale deve attenersi e quindi limitarsi.

${ }^{2}$ Art. 1 della L. 42/1999 "il campo proprio di attività e di responsabilità delle professioni sanitarie ... è determinato dai contenuti dei decreti ministeriali istitutivi dei relativi profili professionali e degli ordinamenti didattici dei rispettivi corsi di diploma universitario e di formazione post-base nonché degli specifici codici deontologici".

${ }^{3}$ Proff. Tavani-Zanchetti in Documento "L'esercizio della Radiologia Diagnostica e della Radioterapia nell'ottica medico-giuridica. Campo delle attività e responsabilità del Tecnico Sanitario di Radiologia Medica" giugno 2014 http://www.tsrm.org/wp-content/uploads/2014/07/Tavani-Zanchetti-firmato-corretto.pdf

${ }^{4}$ prevista dal proprio profilo professionale e dal corso di studi ed inserita nel tirocinio tecnico-pratico

${ }^{5}$ Art. 57, co. 2 DIRETTIVA 2013/59/EURATOM 
radio-protezionistici e qualora, lo possa dimostrare (mediante un'adeguata esperienza pratica, lo specifico aggiornamento professionale e la disponibilità di una opportuna tecnologia e organizzazione) in ulteriori capacità, caratterizzate da particolare difficoltà o, ad esempio, necessarie per tecniche di nuova introduzione. Infatti, in alcuni contesti operativi, per la natura delle prestazioni erogate, al TSRM, è richiesto un profilo di alta qualificazione, come per il caso delle cosiddette "pratiche speciali", comportanti alte dosi per il paziente, e rappresentate, ad esempio, da procedure erogate nell'ambito della cardiologia interventistica o della medicina nucleare, con l'utilizzo di - speciali attrezzature medico-radiologiche, opportune tecniche pratiche e strumenti ausiliari adeguati per le esposizioni mediche, - il ricorso a programmi di garanzia della qualità e di valutazione della dose o di verifica dell'attività somministrata.

La cardiologia interventistica, rappresenta una delle discipline che ha tratto innegabili vantaggi grazie alle attività interventistiche endovascolari, realizzate con l'azione complementare della strumentazione radiologica e opportuni materiali e dispositivi, di sicuro rilievo, raggiungendo, nel corso degli anni, notevoli risultati, anche in pazienti ad elevata complessità clinica e grazie all'evoluzione tecnologica, farmacologica ed organizzativa. La cardiologia interventistica, potendo avvalersi di apparecchiature più performanti, è utilizzata per introdurre e/o guidare, nell'organismo, dispositivi, sofisticati e sempre più miniaturizzati, come guide, cateteri, palloncini o stent (con finalità, diagnostiche e/o terapeutiche). Il TSRM, in questo ambito, nella gestione dell'apparecchiatura e delle tecnologie radiologiche (e non) ad essa collegata, è il professionista indispensabile, in quanto conoscitore di ogni aspetto tecnologico e procedurale utile alla migliore e corretta esecuzione della pratica. In due recenti documenti, la società scientifica italiana di Cardiologia Interventistica GISE, ha riconosciuto al TSRM il ruolo indispensabile e le ulteriori competenze quale secondo operatore nelle procedure diagnostiche e interventistiche cardiache (in regime di elezione ed urgenza), assistendo, al tavolo angiografico, lo specialista Cardiologo interventista (primo operatore). Il TSRM si occupa della realizzazione delle migliori immagini proiettive, della predisposizione, della manipolazione, della gestione, del passaggio all'operatore dei materiali, dispositivi e presidi necessari all'esecuzione della prestazione, cooperando con quest'ultimo, nella somministrazione del mezzo di contrasto (in funzione della prescrizione/ posologia indicata dallo stesso specialista e secondo le acquisizioni scientifiche) che avviene, solitamente, con sistemi automatici, fortemente integrati e cablati con i sistemi angiografici, con moduli di controllo e sensori di sicurezza (rilevamento dell'aria, meccanismi di interruzione e limitazione dei volumi di mdc, registrazione dei volumi iniettati).

La medicina nucleare o imaging molecolare, prevede, per l'esecuzione delle prestazioni l'impiego, previa preparazione/produzione, di radiofarmaci, medicinali che, "quando pronti per l'uso, includono uno o più radionuclidi (isotopi radioattivi), incorporati a scopo sanitario", utilizzati per attività di tipo diagnostico o terapeutico e che rispondono a requisiti di qualità, sicurezza ed efficacia, proteggendo, in questo, il paziente da ogni tipo di esposizione indebita connessa al rischio radiologico ed assicurando, contemporaneamente, la massima efficacia diagnostica e terapeutica del radiofarmaco. Tale conformità è garantita attraverso il costante controllo di qualità delle attrezzature e dei radiofarmaci utilizzati, con personale adeguatamente formato e continuamente aggiornato, secondo quanto definito dal DM 30/03/2005 (in vigore dal 30 giugno 2010) "Norme di buona preparazione dei Radiofarmaci per Medicina nucleare" (NBP-MN), un complesso di indicazioni tecniche, pertinenti l'organizzazione e gestione delle attività relative alla preparazione dei radiofarmaci, sia semplici ${ }^{8}$, che estemporanee e che ha preteso adeguamenti strutturali, organizzativi, gestionali e documentali, implicando che la preparazione ed il controllo di qualità dei radiofarmaci siano effettuate da personale specializzato ed in possesso di tutte le conoscenze necessarie per poter operare in condizioni controllate con sorgenti radioattive non sigillate, rappresentando, il TSRM, la figura più pertinente a tali attività.

Nei casi descritti, la legittimazione ad operare in un campo altamente specialistico, da parte del TSRM, trova giustificata applicazione:

- in quanto "attività pratiche" intimamente connesse con l'espletamento della tecnica radiologia o parte di essa (es. cateterismo cardiaco, clisma opaco, preparazione dei radiofarmaci etc), quale condizione indispensabile per la realizzazione della pratica;

- nell'ambito della formazione e preparazione del professionista, in quanto fondata sull'interpretazione sistematica dei tre riferimenti: profilo professionale, l'ordinamento didattico e il codice deontologico;

- dal presupposto che il TSRM sia stato formato, durante il corso di istruzione di base o post base e/o formazione ECM, con l'acquisizione di conoscenze pertinenti ${ }^{9}$.

Infatti, in ambedue i casi, al TSRM è richiesta l'ulteriore qualificazione in funzione delle "pratiche speciali" 10 , in cui si trova ad operare, nella considerazione che, l'assistenza al tavolo angiografico, come secondo operatore, nel caso della Cardiologia inter-

\footnotetext{
${ }^{6}$ Art. 61 DIRETTIVA 2013/59/EURATOM 5 dicembre 201318 e 51

7 Art. 1 co. 1 lett. e del D. Lgs. 219/2006

http://www.agenziafarmaco.gov.it/sites/default/files/DL_2006_219_0.pdf
}

8 preparazioni derivanti da manipolazioni (miscelazioni, diluizioni, ripartizioni, ecc.) di radiofarmaci forniti "pronti all'uso" da Ditte autorizzate, kit (da ricostruire al momento dell'uso), produzione di radionuclidi mediante generatori (come riportato all'allegato A delle stesse NBP-MN del DM 30/03/2005), la cui preparazione e controllo di qualità dei radiofarmaci sono eseguiti seguendo strettamente le istruzioni del fabbricante, come riportate nell'apposita scheda tecnica allegata, e la cui corretta esecuzione delle procedure è sufficiente a consentire che la qualità del prodotto finale risponda ai requisiti indicato dal produttore.

${ }^{9}$ Documento "Preparazione e somministrazione dei mezzi di contrasto, (c.d. MDC) dei radiofarmaci e radionuclidi. Competenze del TSRM", 2013 - http://www.tsrm.org/wp-content/uploads/2013/01/parere-su-preparazione-e-somministrazione-dei-mezzi-di-contrasto.pdf

10 Art. 61 DIRETTIVA 2013/59/EURATOM 5 dicembre 201318 e 51 
ventistica, la preparazione e la somministrazione di radiofarmaci, nel caso della Medicina nucleare, rientrano tra gli aspetti pratici delle procedure medicoradiologiche ${ }^{11}$ che devono essere svolte, obbligatoriamente da "soggetti abilitati a operare, a tale riguardo, in un campo di specializzazione riconosciuto"12, quali sono i TSRM e come desunto, appunto dal profilo professionale $^{13}$ e dall'ordinamento didattico ${ }^{14}$.

L'attività tipica e caratterizzante del TSRM risiede nello svolgere, in via autonoma, o in collaborazione con altre figure sanitarie, su prescrizione medica, le procedure e gli atti tecnici e sanitari, di sua competenza e che, in particolare, richiedono l'uso di sorgenti di radiazioni ionizzanti, sia artificiali che naturali, di energie termiche, ultrasoniche, di risonanza magnetica nucleare nonché gli interventi per la protezionistica fisica o dosimetrica, e tutte le attività collegate aventi finalità di prevenzione, diagnosi e terapia, sia su materiali biologici sia sulla persona, secondo i contenuti delle norme di riferimento (L. 1103/1965, L. 25/1983, DM 746/1994 e il codice deontologico versione del 2004), in differenti ambiti della diagnostica per immagini, della medicina nucleare, della radioterapia, della fisica sanitaria e della radiologia complementare.

Principalmente il TSRM:

1) si occupa della conduzione tecnica della prestazione, in modo adeguato, secondo regole di buona tecnica, per rispondere al quesito clinico posto dal prescrittore e nel rispetto delle più recenti indicazioni della letteratura scientifica, delle linee guida nazionali ed internazionali e/o dei protocolli operativi preventivamente definiti su base aziendale, in stretta collaborazione con il coordinatore del processo radiologico, per la produzione di un'adeguata ed appropriata informazione diagnostica o del risultato terapeutico;

2) è il diretto garante del principio di ottimizzazione (come previsto dal D. Lgs. 187/2000 e Direttiva Euratom 59/2013), secondo cui "tutte le dosi dovute a esposizioni mediche per scopi radiodiagnostici, di radiologia interventistica, di pianificazione, di guida e di verifica devono essere mantenute al livello più basso ragionevolmente ottenibile compatibili con l'ottenimento dell'informazione medica richiesta"15, attraverso:

a. il corretto impiego delle attrezzature e tecnologie medico-radiologiche a lui affidate ;

b. la realizzazione delle operazioni materiali connesse con l'esposizione medica e qualsiasi altro aspetto correlato (come la valutazione di parametrici tecnici e fisici che condizionano le dosi di radiazione, la calibrazione e la manu- tenzione delle attrezzature, la preparazione e la somministrazione di radiofarmaci, l'elaborazione di immagini $\left.{ }^{16}\right)$;

c. l'opportuno impiego di tutti i dispositivi di radioprotezione, individuale ed ambientale, di paziente ed operatore,

d. l'elaborazione, l'archiviazione e la distribuzione delle immagini realizzate e delle dosi erogate (e registrate, di cui ne assume la piena responsabilità e ne valida l'autenticità);

3) è responsabilmente coinvolto:

a. nel processo di giustificazione dal coordinatore del processo radiologico (in alcuni casi preventivamente codificato, con l'adozione di protocolli), verificando, inoltre, la congruità del quesito clinico posto dal prescrittore in relazione alla prestazione da erogare;

b. effettuando un'anamnesi tecnica finalizzata alla migliore conduzione dell'esame;

c. informando il paziente: 1) sulle modalità di svolgimento della prestazione, al fine di ottenere l'opportuna partecipazione del paziente, nel rispetto delle sue condizioni cliniche; 2) sui rischi derivanti dall'esposizione alle radiazioni ionizzanti in funzione della tecnologia utilizzata ( $\mathrm{so}$ prattutto in presenza di donne in età fertile ed al fine di escludere lo stato di gravidanza).

Nella sua attività rende tracciabili le operazioni compiute attraverso la compilazione di opportune schede di documentazione/valutazione tecnica-radiologica, sia in formato cartaceo che su supporto informatico.

\section{RISULTATI E DISCUSSIONE}

Al TSRM e più in generale alle professioni sanitarie oggi, sono richieste notevoli ed ulteriori competenze, probabilmente fino a qualche tempo inimmaginate, perché fortemente legate all'evoluzione tecnologica e alle introduzioni delle scoperte scientifiche. Non si tratta soltanto di assegnare nuove funzioni, formare per certificare il possesso di nuove competenze, ma di riconoscerle, a livello formale ed economico

Al professionista TSRM che si occupa, in particolar modo degli aspetti pratici ${ }^{17}$ e dell'ottimizzazione ${ }^{18}$ delle pratiche medico-radiologiche, comprese le complementari e la preparazione dei radiofarmaci, è richiesto "un elevato livello di competenze e una chiara definizione delle responsabilità e dei compiti, fondamentali per assicurare un'adeguata protezione dei pazienti sottoposti alle procedure di diagnostica per immagini"19 e nel contempo risultati diagnostici, o terapeutici, adeguati.

Gli aspetti della pratica quotidiana, i dettami della

11 Definizione 64, Art. 4 DIRETTIVA 2013/59/EURATOM 5 dicembre 2013

12 Art. 57 co. 2 DIRETTIVA 2013/59/EURATOM 5 dicembre 2013

13 D.M. 26/9/1994 e Legge n. 25 del 31/1/1983

14 disciplinato nel D.M. 24/7/1996, n.168, Tabella XVIII ter: nella Tabella A dell'ordinamento, che si riferisce agli obiettivi didattici, è previsto, nell'area B, lo "Apprendimento della marcatura di radiocomposti", oltre che della "decontaminazione ambientale"; per l'area F si afferma che lo studente deve acquisire le "competenze necessarie per la preparazione delle dosi"; La Tabella B, dedicata all'addestramento professionalizzante prevede la "partecipazione all'esecuzione tecnica" per acquisire la capacità di "approntare le preparazioni semplici di radiofarmaci”.

15 Art. 56, co. 1 Direttiva Euratom 59/2013

16 Direttiva Euratom 59/2013, Art. 4, co. 1 nr. 64)

17 Art. 57 co. 2, DIRETTIVA 2013/59/EURATOM 5 dicembre 2013

18 Art. 56 co. 4, DIRETTIVA 2013/59/EURATOM 5 dicembre 2013

19 Considerando 29, DIRETTIVA 2013/59/EURATOM 5 dicembre 2013 
legislazione e le indicazioni contenute in raccomandazioni o linee guida, convergono tutti sulla necessità di avere professionisti sanitari specializzati. Nonostante questo e le necessità dell'evoluzione scientifica e tecnologica, le professioni sanitarie, in questi anni, hanno incontro notevoli difficoltà per il loro ulteriore sviluppo e, principalmente, sul riconoscimento delle competenze avanzate, nonostante tali compiti specialistici siano comunque realizzati nella pratica professionale.

Il possesso del titolo di master (introdotto attraverso l' art. 6, co. 1, lettera c, della L. 43/2006), identifica il professionista sanitario, quale "specialista" e contemporaneamente (indirettamente), la sua appartenenza ad una specifica area di interesse scientifico-professionale, perseguendo differenti scopi, come 1) 1' evoluzione professionale, 2) l'adeguamento del sistema a nuovi modelli organizzativi (connotati da una diversa e più ampia modalità di "presa in carico" del paziente); 3) l'adeguamento dei processi assistenziali (che di fatto permettono l'erogazione delle prestazione), 4) l'equiparazione sul piano internazionale; 5) permettere risposte più incisive alle mutate esigenze del contesto sanitario.

Per dare concreta applicazione alle disposizioni previste dalla L. 43/2006 il Contratto collettivo nazionale dell'area del comparto sanità, siglato il 21 maggio 2018, ha rivisitato la materia con particolare riferimento ai professionisti in possesso del master di primo livello per le funzioni specialistiche, istituendo gli incarichi di funzione (organizzativi e professionali), come indicato dall'art. 14 del predetto $\mathrm{CCNL}^{20}$, per lo svolgimento di funzioni con assunzione diretta di elevate responsabilità aggiuntive e/o maggiormente complesse rispetto alle attribuzioni proprie della categoria e del profilo di appartenenza:

a. l'incarico di organizzazione comporta l'assunzione di specifiche responsabilità nella gestione dei processi assistenziali e formativi con autonomia gestionale e organizzativa e che possono richiedere anche l'attività di coordinamento di altro personale,

b. l'incarico professionale, per l'esercizio di compiti aggiuntivi e/o maggiormente complessi, comportanti attività con contenuti di alta professionalità e specializzazione che richiedono significative, elevate ed innovative competenze professionali, rispetto a quelle del profilo posseduto ${ }^{21}$ distinguendo:

- professionista specialista, con il possesso del master specialistico di primo livello, di cui all'art. 6 della legge 43/2006, secondo quanto previsto dall'Osservatorio nazionale per le professioni sanitarie istituito tra Ministero della Salute e Ministero dell'Università;
- professionista esperto, con il possesso di competenze avanzate, derivanti da percorsi formativi complementari o attraverso l'esercizio di attività professionali riconosciute dalle stesse Regioni.

L'Osservatorio delle professioni sanitarie presso il $\mathrm{MIUR}^{22}$ ha approvato nel dicembre 2018, dopo un lungo confronto uno specifico documento ${ }^{23}$ con l'individuazione di un elenco di master universitari specialistici rivolti alle 22 professioni sanitarie con l'intento di riorganizzare l'offerta formativa dai Master specialistici, le cui proposte, si presentavano altamente disomogenee, sia negli percorsi didattici offerti che negli esiti, definendo le conoscenze e competenze professionalizzati da raggiungere attraverso gli specifici percorsi formativi, individuando tre tipologie di master:

- Master Trasversali, rivolti a tutte o parte delle professioni, con contenuti prevalentemente organizzativo-gestionali, didattici e di ricerca, con percorsi didattici unici e la presenza di insegnamenti/CFU dedicati alle specifica area professionale;

- Master interprofessionali, rivolti a due o più professioni, su tematiche cliniche a forte integrazione interprofessionale, prevedendo, oltre ad insegnamenti/CFU comuni tra le professioni, anche insegnamenti/CFU dedicati all'approfondimento di aspetti e competenze specifici per ciascuna professione a cui il master è destinato;

- Master specialistici di ciascuna professione, rappresentano lo sviluppo di competenze specialistiche di ogni professione, offrendo, in alcuni casi la "certezza di spendibilità operativa" 24 , per altri la certificazione, attraverso il sistema universitario, di competenze avanzate.

Un esempio interessante, su questa linea, è rappresentato dalla Regione Veneto che ha istituito, con apposito provvedimento ${ }^{25}$, specifiche aree di intervento comportanti compiti aggiuntivi e/o maggiormente complessi per le professioni sanitarie e $\mathrm{i}$ rispettivi requisiti formativi complementari (post diploma), da possedere per poter svolgere attività che richiedono competenze professionali innovative, elevate e significative, rispetto a quelle contemplate dal profilo di base, secondo le esigenze organizzative e di intervento necessarie a livello regionale, riconoscendo, in questo, l'alto contributo di tutte le professioni sanitarie. L'art. 4 della L. 3/2018, meglio nota come "Legge Lorenzin", modificando i capi I, II e III del Decreto legislativo del Capo provvisorio dello Stato 13 settembre 1946, n. 233, ha inteso riordinare la disciplina degli Ordini delle professioni sanitarie. Un intervento atteso da anni, soprattutto per quelle professioni sanitarie che pur regolamentate, con propri profili professionali, non erano mai state ordinate, al pari di quanto

\footnotetext{
${ }^{20}$ Art. 14 CCNL comparto sanità Triennio 2016-2018 https://www.aranagenzia.it/attachments/article/9016/ CCNL\%20comparto\%20SANITA\%20definitivo_sito\%20.pdf

${ }_{21}$ Tenendo conto del dettato di cui all'art. 6 legge 43/2006, decreti istitutivi dei profili professionali (ex co. 3 art. 6 D. Lgs. n. 502/1992)

${ }^{22}$ Ricostituito con apposito Decreto Interministeriale (Salute ed Università) 10 marzo 2016 n. 155

${ }^{23}$ https://www.miur.gov.it/web/guest/-/universita-90-nuovi-master-per-le-22-professioni-sanitarie

24 ai fini dell'art. 16 comma 7 del CCNL

${ }_{25}$ Pubblicazione Bur Veneto n. 127 del 08 novembre 2019 https://bur.regione.veneto.it/BurvServices/Pubblica/DettaglioDgr. aspx?id $=406935$
} 
avveniva per le professioni di Infermiere, Assistente sanitario, Ostetrica e Tecnico sanitario di radiologia medica, già organizzate in collegi professionali circoscrizionali, corrispondenti alle provincie geografiche. È stato così costituito l'Ordine dei tecnici sanitari di radiologia medica e delle professioni sanitarie tecniche della riabilitazione e delle prevenzione, che in tanti, sui social e sui media, abbreviano con i termini di "maxi-Ordine" o "Ordine multi-Albo".

Ai sensi dell'art. 1 del Decreto del Ministero della Salute del 13/03/2018 sono stati istituiti, presso i neocostituiti Ordini, oltre l'Albo dei tecnici sanitari di radiologia medica e l'Albo degli Assistenti sanitari, in rappresentanza delle altre 17 professioni ad esso afferenti, altrettanti Albi, con l'obbligo d'iscrizione per l'esercizio professionale, in qualunque forma giuridica svolto (co. 2 art. 5 D. Lgs. CPS 233/1946 come modificato dall'art. 4 della L. 3/2018), garantendo un sistema completamente regolamentato per $i$ professionisti e per $i$ cittadini, in cui soltanto chi è iscritto al relativo Albo professionale (o in uno degli Elenchi speciali ad esaurimento), ha titolo ad operare e ad esercitare la professione, con la possibilità per il cittadino di conoscere, attraverso il portale dedicato (con una procedura dematerializzata e telematica per le iscrizioni), se colui che, in una struttura pubblica o privata, in regime di dipendenza o di libera professione, lo ha preso in carico, è un professionista titolato ad operare, perché sottoposto al controllo e alla verifica da parte dell'Ordine.

La riforma, così operata, ha attribuito agli Ordini alcune importanti funzioni con il fine di tutelare gli interessi pubblici garantiti dall'ordinamento dello Stato e connessi con l'esercizio professionale.

Gli ordini e le relative Federazioni nazionali sono enti pubblici non economici che agiscono quali organi sussidiari dello Stato, secondo autonomia patrimoniale, finanziaria, regolamentare e disciplinare e sottoposti alla vigilanza del Ministero della salute. Le attività sono rivolte a promuovere ed assicurare l'indipendenza, l'autonomia, la responsabilità delle professioni e dell'esercizio professionale, la qualità tecnico-professionale, la valorizzazione della funzione sociale, garantendo e tutelando la salute individuale e collettiva.

Spetta, inoltre, all'Ente, verificare il possesso, da parte dei professionisti, del titolo professionale abilitante, senza il quale si configura il reato di esercizio abusivo della professione stessa, secondo quanto disposto dall'art. 12 della L. 4/2018, curando la tenuta, e la pubblicità degli albi dei professionisti e, laddove previsti dalle norme, degli elenchi speciali. Inoltre l'Ordine ed ogni suo rispettivo Albo, a cui spetta la rappresentanza esponenzialmente di ogni singola professione, (ai sensi dell'art. 2 D. Lgs. CPS 233/1946 per gli Ordini comprendenti più professioni) promuove e favorisce iniziative funzionali per il progresso culturale degli iscritti, anche in riferimento alla formazione e fornisce, alle autorità locali, il proprio contributo per lo studio e l'attuazione dei provvedimenti che interessano la professione.

Le funzioni ed il ruolo strategico che la riforma operata attribuisce agli Ordini, risultano fondamentali per la realizzazione di interventi, nazionali e locali, utili all'evoluzione professionale, adeguando e migliorando il livello culturale, deontologico e professionale, degli esercenti le professioni.

In conclusione possiamo sostenere che il ruolo del TSRM, è già cambiato, complici le notevoli esigenze imposte dall'evoluzione scientifica e tecnologica che hanno caratterizzato, in questi anni, il mondo della diagnostica per immagini. Abbiamo più volte accennato, infatti, "alla continua evoluzione della pratica clinica, in cui le tecnologie hanno assunto una crescente rilevanza, divenendo parte imprescindibile della prestazione stessa. Un corretto approccio diagnostico e terapeutico non può infatti prescindere dalla disponibilità di un adeguato supporto tecnologico. Inoltre, le attrezzature più obsolete hanno impatto sulla spesa sanitaria in termini di costi di manutenzione, tendenzialmente alti, generalmente a fronte di bassa redditività e minore efficienza produttiva"26. Su questo aspetto è utile segnalare che, in Italia, secondo i dati di Assobiomedica ${ }^{27}$ e di alcuni studi condotti dallo stesso Ministero della Salute ${ }^{28}$ un gran numero di apparecchiature ha ormai superato i limiti dell'obsolescenza tecnologica e non è più in grado di soddisfare gli standard di utilizzo. Secondo tali studi, l'età media dei sistemi di diagnostica per immagini è superiore a 7 anni, con punte addirittura di oltre i 13 anni nel caso di alcune tecnologie.

La rilevanza assunta dalle tecnologie non può considerarsi separata dal livello di competenze di chi tali tecnologie le deve far funzionare ed utilizzare per l'erogazione delle prestazioni.

L'esecuzione di una semplice radiografia è passata da elementari tecniche di sviluppo della pellicola radiografica, all'applicazione di sofisticati sistemi di gestione digitale delle immagini, con la possibilità, ad esempio di applicare filtri e finestre di visualizzazioni, misurazioni ed annotazioni, oppure di trasferire a distanza ed archiviare in sistemi cloud le immagini, in formato dematerializzato, richiamandole quando necessario. Le semplici acquisizioni tomografiche delle "TAC" hanno lasciato il campo a scansioni volumetriche che in pochi secondo, ad esempio, sono nelle condizioni di ricostruire l'albero coronarico, il decorso dei vasi periferici o lo studio dell'aorta, permettendo ricostruzioni di volume rendering. Alle conoscenze classiche "nell'uso di sorgenti di radiazioni ionizzanti sia artificiali che naturali, delle energie termiche, ultrasoniche, di risonanza magnetica nucleare, nonché gli interventi per la protezionistica fisica o dosimetrica" 29 , comportanti "la valutazione di parametrici tecnici e fisici che condizionano le dosi di radiazione, la calibrazione e la

26 Ministero della Salute, documento "Rilevazione della apparecchiature sanitarie in Italia Flusso informativo per il monitoraggio delle apparecchiature sanitarie in uso presso le strutture sanitarie pubbliche, private accreditate e private non accreditate Rapporto 2017 " p. 17 http://www.salute.gov.it/imgs/C_17_pubblicazioni_2678_allegato.pdf

27 In “STUDI”, nr. 38 - novembre 2017 “OSSERVATORIO PARCO INSTALLATO: LE APPARECCHIATURE DI DIAGNOSTICA PER IMMAGINI IN ITALIA EDIZIONE 2017” https://www.quotidianosanita.it/allegati/allegato195048.pdf

28 Ministero della Salute - Rapporto sulla rilevazione delle apparecchiature sanitarie in Italia anno 2017

http://www.salute.gov.it/imgs/C_17_pubblicazioni_2678_allegato.pdf

29 DM 746/1994 e L. $\underline{25 / 1983, ~}$ 
manutenzione delle attrezzature, la preparazione e la somministrazione di radiofarmaci, l'elaborazione di immagini"30 si sono aggiunte le dovute conoscenze digitali, teoriche e pratiche connesse, con l'utilizzo di nuovi sistemi radiologici.

Come già evidenziato, lo sviluppo tecnologico, che ha ampliato le conoscenze, ha introdotto, all'interno dell'ampio sistema della diagnostica per immagini, alcune sub specialità che richiedono, necessariamente, l'acquisizione di specifiche competenze teoriche e, soprattutto, pratiche, accompagnate da una formazione "sul campo".

30 Direttiva Euratom 53/2019, Art. 4, co. 1 nr. 64

\section{REFERENCES}

1. A. Di Lascio, 2019, "Strumenti per l'ottimizzazione della dose nella preparazione e semministrazione dei radiofarmaci in medicina nucleare" Napoli, in: "Journal of advanced health care", pubbl. online, Settembre 2019

2. A. Di Lascio, 2019, "La formazione continua in medicina: da obbligo di legge ad opportunità. Gli strumenti a disposizione del professionista per il nuovo triennio formativo 2020-2022”, Napoli, in Giornalesanità.it - Professionisti della salute al servizio del cittadino - del 7 gennaio 2020

3. A. Di Lascio, 2019, "La consulenza tecnica nei giudizi di responsabilità sanitaria", Roma In: "eHealth" (ISBN 20384238), a. XI, n. 72, 2019 nov/dic - pp. 14-25

4. A. Di Lascio, 2019, “Tecnico sanitario di radiologia medica: È cambiato il ruolo dei professionisti”, Napoli in Giornalesanità.it - Professionisti della salute al servizio del cittadino - del 19 dicembre 2019

5. A. Di Lascio, 2019, "Responsabilità professionale e qualità delle cure: una nuova vision per le professioni sanitarie", Napoli, in Giornalesanità.it - Professionisti della salute al servizio del cittadino - del 22 novembre 2019

6. A. Di Lascio, 2019, "Elezioni Commissioni d'Albo: un opportunità per le professioni sanitarie dell'Ordine Tsrm Pstrp", Napoli, in quotidianosanità.it - Quotidiano on line di informazione sanitaria - del 16 novembre 2019

7. A. Di Lascio, 2019, "Commissioni d'Albo: al voto per le professioni sanitarie. Norme e leggi, vademecum per l'urna" in Giornalesanità.it - Professionisti della salute al servizio del cittadino - del 15 novembre 2019

8. A. Di Lascio, 2019, "Lo sviluppo della tecnologia digitale”, Roma, In: "eHealth" (ISBN 2038-4238), a. XI, n. 71,2019 set/ott - pp. 26-33

9. A. Di Lascio, F. Rossetti, 2016, "La responsabilità professionale. Strumenti a tutela del professionista", Napoli in Radiologia \& Futuro (2016) XIV (3) 912-918;

10. A. Di Lascio, C. Vazzana, F. Rossetti, 2017, “Tutela della Responsabilità professionale dei TSRM”, Roma in e-Health (2017) nr. 53 37-56

11. A. Di Lascio, 2018, "Ripensare la Sanità ai tempi del digitale" a cura di G. Cosentino, Associazione Scientifica Sanità Digitale (Roma), autore del capitolo "Telemedicina in radiologia. Il Tecnico Sanitario di Radiologia Medica, aspetti operativi e di sicurezza”(pp. 133 - 143), autore Youcanprint-borè srl - ISBN 9788827848807

12. L. 42/1999

13. M. Tavani, L. Zanchetti, 2014, Documento "L'esercizio della Radiologia Diagnostica e della Radioterapia nell'ottica medico-giuridica. Campo delle attività e responsabilità del Tecnico Sanitario di Radiologia Medica";

14. L. Fiorillo, 2019, "Il diritto del lavoro nel pubblico impiego", Padova: ed. Piccin;

15. Piccioli, A. Sarteanesi, 2017, "La Responsabilità nelle professioni sanitarie”, Milano: ed. Giuffrè;

16. M. L. Missiaggia, 2017, "La responsabilità sanitaria. Guida operativa alla riforma Gelli (L. 8 marzo 2017, n. 24)", Piacenza, ed. La Tribuna;

17. A cura di F. Volpe, 2018, "La nuova responsabilità sanitaria dopo la riforma Gelli-Bianco (legge n. 24/2017)", Torino, ed. Zanichelli;

18. AA.VV., 2005, “La responsabilità professionale del medico", Santarcangelo di Romagna RN, ed. Maggioli;

19. M. Di Pirro, 2018, "La nuova responsabilità medica e il risarcimento del danno", Napoli, ed. Simone;

20. A cura di C. Consolo, 2018, "Il contenzioso sulla nuova responsabilità sanitaria (prima e durante il processo), Torino, ed. G. Giappichelli;

21. AA.VV., 2019, "La gestione della responsabilità civile sanitaria: tra risk management e assicurazione", Piacenza, ed. La Tribuna;

22. DIRETTIVA 2013/59/EURATOM

23. CCNL comparto sanità Triennio 2016-2018

24. L. $43 / 2006$,

25. Lgs. n. 502/1992

26. NBP-MN del DM 30/03/2005)

27. L. $3 / 2018$

28. L. $1103 / 1965$,

29. L. $25 / 1983$

30. D. Lgs. $219 / 2006$

31. DM 746/1994

32. codice deontologico TSRM versione del 2004 